\title{
Gestión de la Seguridad y Salud en el Trabajo
}

César A. Chávez Orozco

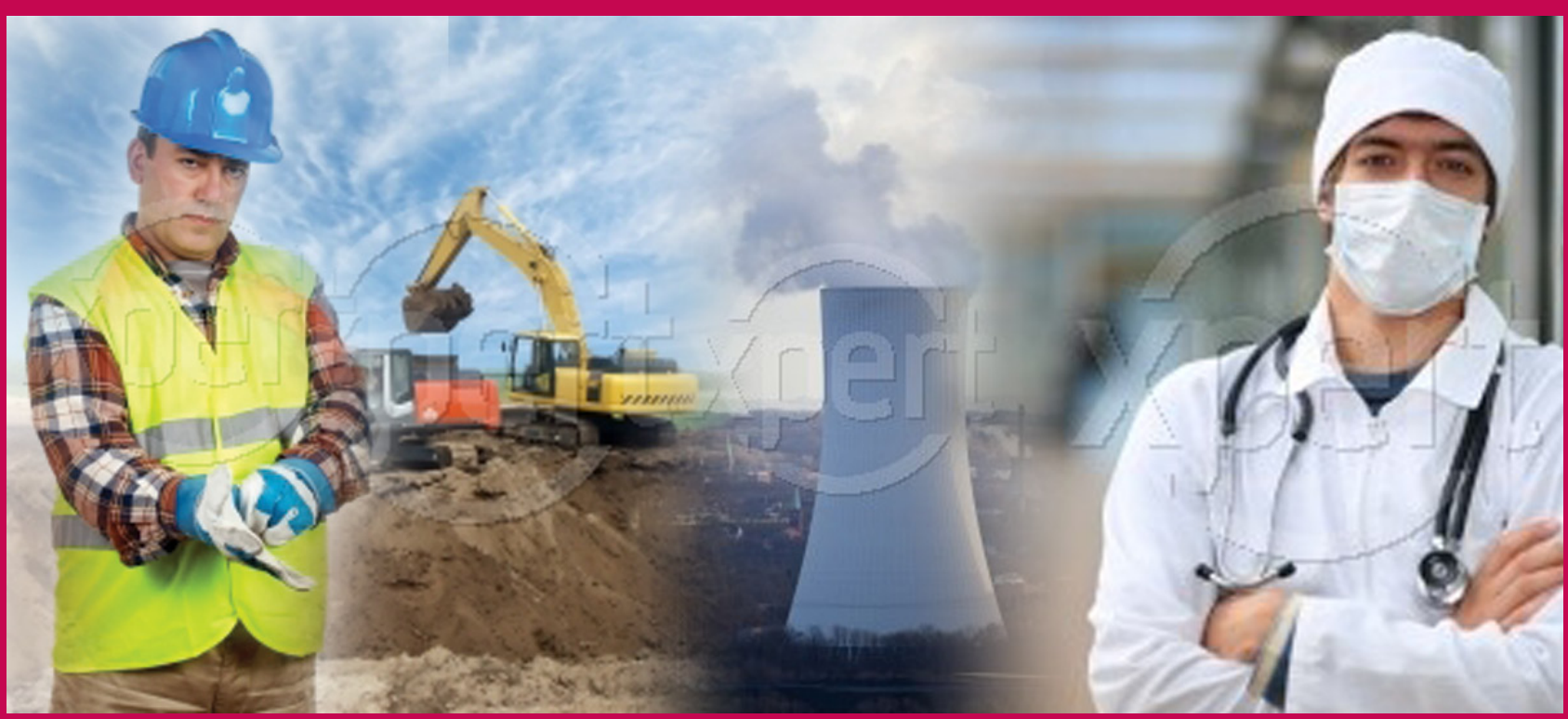

Resumen - El presente trabajo tiene como propósito realizar un breve análisis de la presencia de los riesgos en los lugares de trabajo, su relación con la salud y la importancia de su control para garantizar la protección de la salud de los trabajadores y por ende de los bienes y recursos de la organización. Las exigencias legales cada día más estrictas, los requerimientos de competitividad y las exigencias de los trabajadores, hacen que día a día se incremente la necesidad de que las empresas gestionen sus riesgos.

La prevención no trata solamente de defender al trabajador frente a la amenaza del riesgo laboral, sino que trata de procurarle un resultado positivo de promoción de su salud e integridad, en definitiva, de su desarrollo personal.

El avance de la prevención que se manifiesta en los tiempos actuales como un salto hacia nuevas fronteras, ha promovido la integración de la gestión de la seguridad y salud en el trabajo en toda la gestión integral de la organización. Para conseguir esta integración, se han establecido varias herramientas que permiten realizar las actividades de prevención orientando siempre a la mejora continua.

Los sistemas de gestión de seguridad y salud laboral más difundidos en el Ecuador son: el sistema de administración de la seguridad y salud en el trabajo, promovido por la entidad aseguradora del país (IESS) y el estándar internacional OHSAS 18001:2007, promovido por el creciente interés de las empresas de disponer sistemas certificados.

1 César A. Chávez Orozco, 097488427, coca2550@ute.edu.ec
Para la aplicación de estos modelos de gestión, es importante que la organización se vea como un sistema integral, en donde los procesos preventivos formen parte integrante de todos los procesos de la organización.

Palabras clave- Gestión de riesgos, Gestión de seguridad y salud, Prevención de riesgos.

\section{Introducción}

El trabajo constituye para el ser humano, por una parte, un medio para garantizar su supervivencia, desarrollar sus potencialidades físicas, intelectuales y sociales; y conseguir un mejor estilo de vida; por otra parte, constituye un derecho y un deber de todo ciudadano.

Pero, para poder desempeñar las exigencias de este trabajo, el ser humano hace uso de todas sus capacidades, poniendoenjuegosubien más preciado, su salud;entendiéndose como Salud, el estado de bienestar físico, psíquico y social del individuo y no únicamente la ausencia de enfermedad.

Podría decirse, que el trabajo y la salud se encuentran íntimamente relacionados, puesto que el trabajador necesita estar sano para poder realizar el trabajo; y por otra parte el trabajo, con todos sus componentes y variables, se transforma en una fuente de riesgo para su salud.

En una primera aproximación, se puede decir que en el binomio trabajo-salud, se produce una relación en ambas direcciones, sea dando lugar a efectos positivos o negativos. De una manera simplificada se podría dividir 
ambos fenómenos en dos categorías, de buena y mala salud, frente a buenas y malas condiciones de trabajo. En la Tabla 1 se presentan algunos ejemplos de esta realidad multidimensional.

\begin{tabular}{|c|l|l|}
\hline & Salud Positiva & \\
\hline $\begin{array}{c}\text { Trabajo } \\
\text { Positivo }\end{array}$ & $\begin{array}{l}\text {-Salud y bienestar } \\
\text { Productividad y calidad }\end{array}$ & Incapacidad \\
\hline $\begin{array}{c}\text { Trabajo } \\
\text { Negativo }\end{array}$ & $\cdot$ Insatisfacción & \\
\hline
\end{tabular}

Tabla 1. Relación multidimensional entre el trabajo y la salud

\section{Gestión de la Seguridad y Salud en el Trabajo}

La Seguridad y Salud Laboral, se puede definir como "las condiciones y factores que inciden en el bienestar de los trabajadores, contratistas, visitantes y otras personas en el sitio de trabajo".

La seguridad y salud laboral, más allá de ser una estrategia enfocada simplemente a la prevención de lesiones y enfermedades, es un concepto que ayuda a la competitividad de las empresas; fundamentado al considerar que los accidentes y enfermedades como una consecuencia de la ineficiencia de los procesos, de quienes los ejecutan y de las tecnologías que se utilizan al interior de la empresa, que a su vez depende de su estructura y capacidad económica. En la medida en que se actúen sobre las causas que ocasionan dichas ineficiencias, mediante la aplicación de medidas preventivas, se generarán ahorros en materia prima, insumos, energía, impactos al medio ambiente, y; lo más importante, se evitará el sufrimiento y dolor que provoca un accidente o una enfermedad laboral. De esta forma se contribuirá a mejorar la capacidad competitiva de la empresa y también su desempeño laboral.

Los procesos de globalización están cambiando rápidamente el contexto de las empresas, con relación a su gestión, siendo muy importante, la Gestión de la Seguridad y Salud laboral. Se incrementan nuevas exigencias de los mercados relacionados con el cumplimiento de estándares de seguridad, haciendo prever que en un futuro muy cercano, se convertirá en un aspecto de supervivencia empresarial.

El sistema de Gestión de SSO es parte del sistema de gestión total de la organización, que facilita la administración de los riesgos laborales asociados con sus procesos.

La característica principal de los sistemas de gestión, es que basan su acción en el ciclo de mejoramiento continuo P-H-V-A (Planificar - Hacer - Verificar - Actuar), presentado por Deming a partir del año 1950.

Los resultados de la implementación de este ciclo permiten a las empresas una mejora integral de la competitividad, de los productos y servicios, mejorando continuamente la calidad, reduciendo los costes, optimizando la productividad, reduciendo los precios, incrementando la participación del mercado y aumentando la rentabilidad de la empresa.

"Planificar": Establecer los objetivos y procesos necesarios para conseguir resultados de acuerdo con los requisitos de las partes interesadas y las políticas de la organización;

"Hacer": Implementar los procesos, planes, programas;

"Verificar": Realizar el seguimiento y la medición de los procesos, planes, programas respecto a las políticas, los objetivos y los requisitos de las partes interesadas, e informar sobre los resultados.

"Actuar": Tomar acciones para mejorar continuamente el desempeño de los procesos y del sistema.

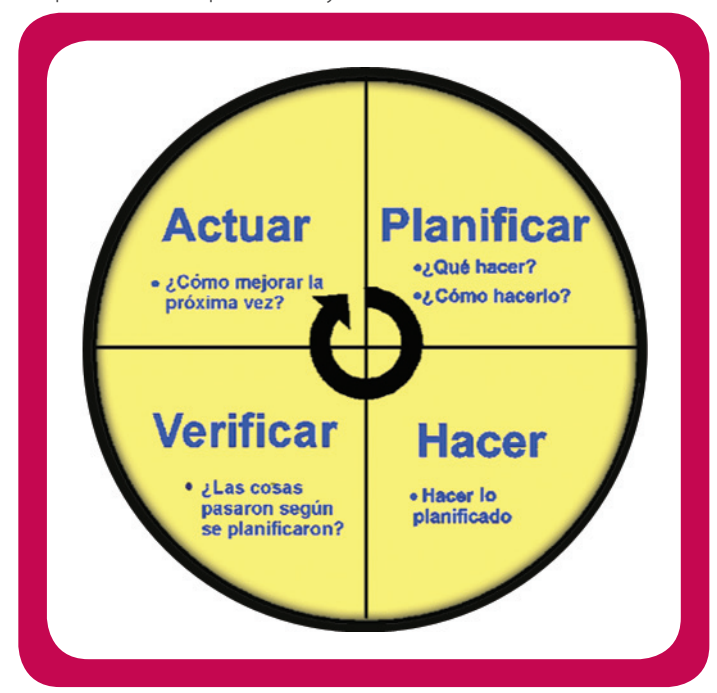

Figura 1. Ciclo de mejora continua o de Deming

Para comprender la generación de valor proporcionado por la gestión de la seguridad y salud, es necesario comprender como funciona una empresa, no desde el punto de vista clásico, sino desde los actuales planteamientos empresariales.

La empresa debe ser entendida como un sistema (es decir, una entidad con sentido completo, compuesta por una serie de unidades relacionadas entre sí, cuyo resultado es mayor que la suma de las partes y en el que cada unidad tiene sentido en sí misma), formando un conjunto de procesos encaminados a generar valor $y$, en conjunto, a entregar el producto o servicio más adecuado a las necesidades de los clientes. Dentro de los procesos que integran la empresa, algunos estarán al servicio del cliente interno: el trabajador. Un fallo en alguno de los 
procesos va a incidir en el resultado del sistema, lo que en mayor o menor medida incidirá en el resultado de la empresa. Por esta razón, el fallo en un proceso preventivo no sólo va a significar, un riesgo para la salud del trabajador, sino que a su vez genera un riesgo para el resultado final de la empresa, afectando su rentabilidad.

La empresa vista como un todo, como un sistema adaptativo en el que aparecen unas entradas (inputs) se transforman en salidas (outputs), incorpora el elemento clave que justifica la razón de ser de la organización: la existencia de un cliente final y de clientes intermedios que se relacionan por los procesos que soportan sus actividades y que están alineados para satisfacer sus necesidades.

La integración de la prevención como un conjunto de procesos internos dirigidos a la mejora de las condiciones de trabajo, no sólo redundará en una mejor seguridad y salud, sino también en mejores resultados empresariales; por lo tanto, es importante que los empresarios inviertan en los procesos preventivos de igual forma como invierten en los procesos productivos.

El sistema de gestión de seguridad y salud es la forma en que la empresa lleva a cabo sus actividades preventivas, definiendo la organización, las funciones y responsabilidades dentro de los miembros de la empresa, las actividades que se deben llevar a cabo y la documentación que soporta todo ello.

Se han desarrollado algunos modelos para la Gestión de los Riegos Laborales, siendo los más conocidos y aplicables en el Ecuador el Sistema de Administración de la Seguridad y Salud en el Trabajo, diseñado por el Instituto Ecuatoriano de Seguridad Social, establecido como una exigencia legal; y el estándar OHSAS 18001:2007, como un sistema voluntario.

\section{Sistema de Administración de la Seguridad y Salud en el Trabajo}

El Sistema de Administración de la Seguridad y Salud en el Trabajo, es un modelo de gestión establecido por la Dirección del Seguro General de Riesgos del Trabajo del Instituto Ecuatoriano de Seguridad Social (IESS) el mismo que centra su objetivo en la prevención de riesgos laborales. Involucra la gestión técnica, la gestión administrativa y la gestión del talento humano, que deben formar parte de la política y el compromiso de la gerencia en beneficio de la salud y la seguridad de los trabajadores, el desarrollo y productividad de las empresas y de toda la sociedad.

\section{Gestión Administrativa}

En este módulo se integra todo el conjunto de políticas, estrategias y acciones que determinan la estructura organizacional, asignación de responsabilidades y el uso de recursos, en los procesos de planificación, implementación y evaluación de la seguridad y salud; este requisito está compuesto por capítulos:

- Política

- Organización

- Planificación de la SST

- Implementación del Plan

- Evaluación y seguimiento.

\section{Gestión del Talento Humano}

Aquí, se busca descubrir, desarrollar, aplicar y evaluar los conocimientos, habilidades, destrezas y comportamientos del trabajador; orientados a generar y potenciar el capital humano, que agregue valor a las actividades organizacionales y minimice los riesgos del trabajo; contempla los siguientes aspectos:

- Selección

- Información

- Formación y capacitación

- Comunicación

\section{Gestión Técnica}

Considera los sistemas normativos, herramientas y métodos que permiten identificar, conocer, medir y evaluar los riesgos del trabajo; $y$, establecer las medidas correctivas para prevenir y minimizar las pérdidas organizacionales por un deficiente desempeño, está compuesto por los siguientes capítulos:

- Identificación objetivo.

- Identificación subjetiva

- Medición

- Evaluación ambiental, biológica y psicológica

- Principios de acción preventiva

- Vigilancia de la salud de los trabajadores.

- Seguimiento.

- Actividades proactivas y reactivas básicas

- Investigación de accidentes e incidentes

- Programa de mantenimiento preventivo, predictivo y correctivo.

- Programa de inspecciones planeadas.

- Planes de emergencia y contingencia.

- Equipos de protección personal.

- Registros del Sistema de Administración de SST.

- Auditorías

- Reglamento interno de seguridad y salud en el trabajo. 


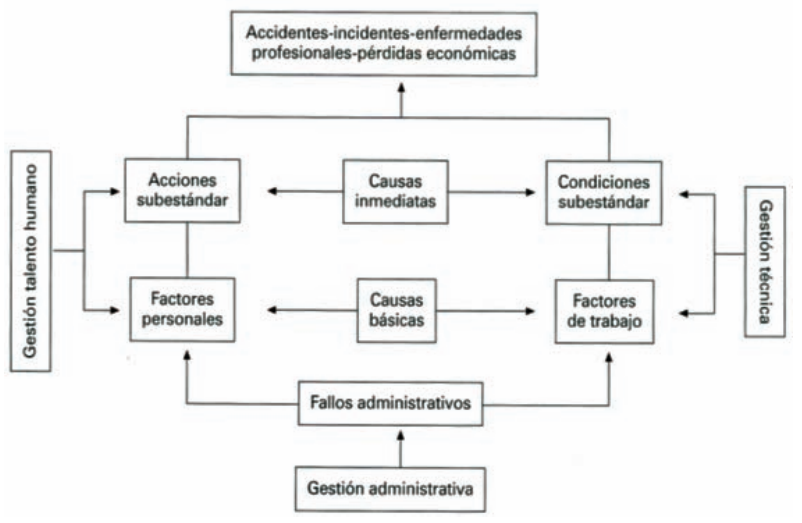

Figura 2. Sistema de Administración de la Seguridad y Salud en el Trabajo

\section{Norma OHSAS 18001:2007}

Este estándar proporciona los elementos de un sistema de gestión de la Seguridad y Salud en el Trabajo, que puede ser integrado con otros requisitos de gestión, para ayudar a las organizaciones a lograr los objetivos de Seguridad y Salud en el Trabajo y económicos.

La implementación de este estándar permitirá a una organización, desarrollar e implementar una política y unos objetivos que tengan en cuenta los requisitos legales y la información sobre los riesgos para la seguridad y salud de los trabajadores. A través de éste, se hace énfasis en las prácticas proactivas preventivas, mediante la identificación de peligros y la evaluación y control de los riesgos en el sitio de trabajo.

La política es el punto inicial y crucial para la implementación del sistema de gestión. Contiene un sentido general de dirección y se fijan los principios de acción para la organización. Determina los objetivos respecto a la responsabilidad y desempeño de Seguridad y Salud Ocupacional requeridos en toda la organización y demuestra el compromiso formal de la organización, particularmente el de su alta gerencia, con la buena gestión del sistema.

\section{Planificación}

Este aspecto tiene que ver con la estrategia para el desarrollo del sistema, incluye la identificación de peligros, así como la evaluación y control de riesgos, de sus actividades, productos y servicios. También contempla la identificación de los requisitos legales y normativos que son aplicables para la organización, en materia de seguridad y salud ocupacional y el establecimiento de objetivos medibles, para poder cumplir con lo especificado en la política.

\section{Implementación y Operación}

Contempla la estructura administrativa que permita la implantación del sistema, además del suministro de los recursos necesarios para el mismo. Incluye requisitos para el entrenamiento, concientización y competencia de los empleados y contratistas, la participación, consulta y comunicación con las diferentes partes interesadas; la documentación que soporta el sistema y su control, así como el control operativo y la preparación y respuesta ante

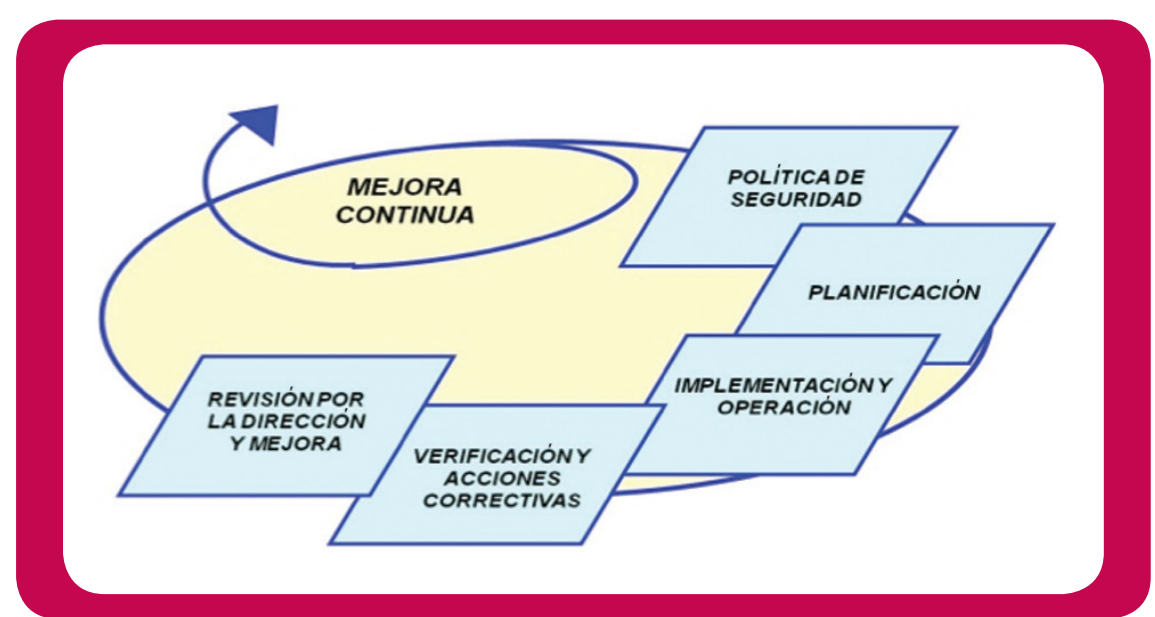

emergencias.

Verificación y acción correctiva y preventiva

Este capítulo permite monitorear el desempeño del sistema de gestión de sSO para determinar su cumplimiento; el establecimiento de procedimientos para el reporte y la evaluación/investigación de incidentes y no conformidades para prevenir la ocurrencia de sucesos similares y detectar causas potenciales de no conformidades; la conservación de registros para demostrar que el

Figura 3. Modelo de sistema de gestión de la Seguridad y Salud en el Trabajo para el estándar OHSAS 18001.

\section{Política de Seguridad y Salud Ocupacional}

Una política de Seguridad y Salud Ocupacional es un conjunto de principios e intenciones formales y documentadas en relación con la seguridad y salud de los trabajadores. sistema de gestión opera de manera efectiva y que los procesos se han llevado a cabo bajo condiciones seguras; así mismo se contempla la auditoría como herramienta de revisión y evaluación continua de la efectividad del sistema de gestión.

\section{Revisión por la Gerencia}

La revisión de sistema es el proceso mediante el cual 
la dirección de la empresa, basándose en los resultados de la auditoría, la información recibida a través de los controles y el análisis del cumplimiento y resultado de los objetivos, analiza si el sistema es adecuado para el cumplimiento de la política de seguridad y salud, decidiendo las modificaciones y mejoras que requiera, asegurando de esta forma la mejora continua.

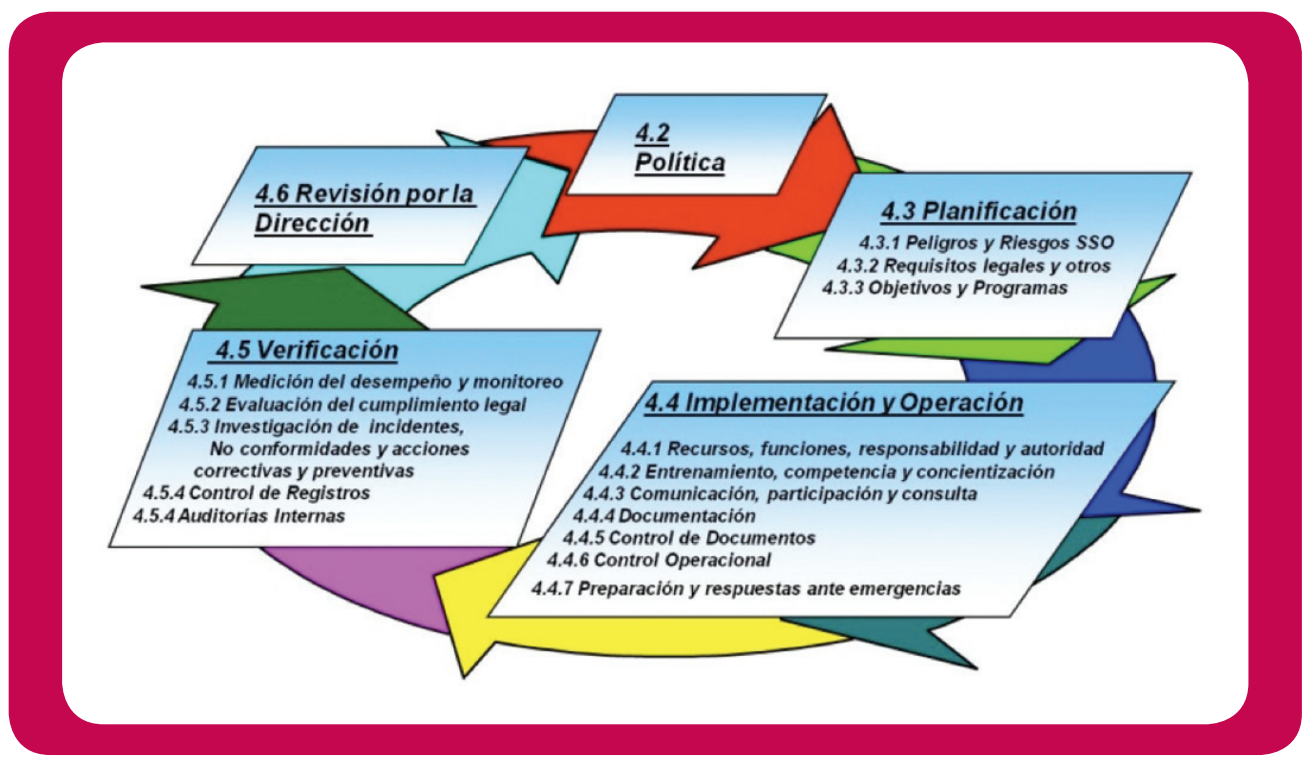

Figura 3. Modelo de sistema de gestión de la Seguridad y Salud en el Trabajo para el estándar OHSAS 18001

Finalmente, la gestión de la seguridad y salud, como cualquier otra actividad empresarial, debe analizarse dentro de una cuenta de resultados. Es preciso demostrar que los beneficios de la inversión en prevención no sólo son morales, sino que, además, mejoran la cuenta de resultados de la empresa. Se podría decir que no hay gestión eficaz si no es eficiente, es decir, si no tiene en cuenta, además del resultado, el costo para llegar a él.

\section{Bibliografía}

- A. Beltrán, M. Bernal, y S. A. Garcia, "La norma OHSAS 18001 y su implementación," ICONTEC, pp.9-11, 2007.

- AENOR, "Sistemas de gestión de la seguridad y salud en el trabajo - Requisitos," AENOR, 2007

- G. Gómez, "Manual para la formación en prevención de riesgos laborales,"Ecoiuris, pp. 25-57.

- C. Ruiz, A. M. García, J. Delclós y F. G. Benavides, "Salud Laboral", cap. 3, 14, 18, Mason, 2007.

- J. M. Cortéz Díaz, "Seguridad e Higiene del Trabajo, Cap. 1, Tébar, 2007.

- C. Ramírez Cavassa, "Seguridad Industrial", pp 13 - 20, Limusa, 2008.

- IESS,"Sistema de Administración de la Seguridad y Salud en el Trabajo", pp. 21-42, Quito, 2007

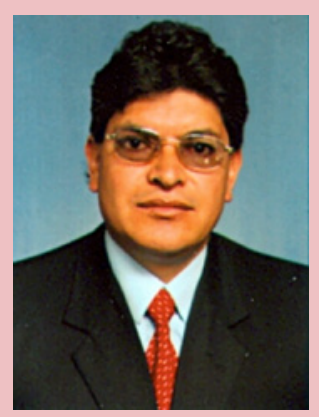

Autor. César A. Chávez Orozco

Nació en Riobamba, el 2 de Septiembre de 1966. Se graduó en la Escuela Superior Politécnica de Chimborazo en Ingeniería Mecánica; Universidad Central del Ecuador, Magister en Seguridad, Salud y Ambiente. Posee conocimientos adicionales como Auditor Líder OHSAS 18001, Auditor Interno en ISO 9001, ISO 14001 y OHSAS 18001. Tiene certificaciones como: Oficial Certificado en Protección CPO (Certified Protection Officer) y Certificación en Gerencia y Supervisión de Protección CSSM (Certification in Supervision \& Security Management). Ha realizado trabajos como diseño e implementación de sistemas de gestión de seguridad y salud ocupacional, elaboración y aprobación de Reglamentos Internos de Seguridad y Salud en el Trabajo; Análisis de Riesgos.

Ejerce profesionalmente en las áreas de Seguridad y Salud Ocupacional y Medio Ambiente en el Cuerpo de Ingenieros del Ejército. Es docente de posgrado en la Universidad Tecnológica Equinoccial, Universidad Central del Ecuador, Universidad Nacional de Chimborazo 